\title{
Economías de guerra: algunas ideas sobre la importancia de la Primera Guerra Mundial desde el punto de vista económico
}

War economies: some thoughts on the importance of the First World War from

the economic point of view

\section{Vicente Guerrero Flores*:}

"La guerra mundial tenía que producirse, pero nadie, ni siquiera el más cualificado de los profetas, comprendían realmente el tipo de guerra que sería. Y cuando finalmente el mundo se vio al borde del abismo, los dirigentes se precipitaron en él sin dar crédito a lo sucedía"

Hobsbawn, 2013

\section{Resumen}

El objetivo esencial de este ensayo es analizar cómo los países participantes en la Primera Guerra Mundial pagaron el esfuerzo bélico y sus consecuencias económicas, especialmente las reparaciones de guerra. Ambos requieren grandes cambios estructurales en la producción, en la política económica y en las relaciones entre agentes productivos internos y externos

$$
\begin{array}{ll}
\text { Palabras clave: } \\
\text { • } \quad \text { Primera Guerra Mundial } \\
\text { - } \quad \text { Reponomías de guerra } \\
\end{array}
$$

\begin{abstract}
The main objective of this paper is to analyze how the countries participating in World War I paid the war effort and its consequences, specially war reparations. Both require major structural changes in production, economic policy and the relationship between internal and external agents
\end{abstract}

\section{Keywords: \\ World War I \\ - Economies of War \\ - War Reparations}

JEL: N440, F34, N240, H63

\section{Introducción}

El centenario de la Primera Guerra Mundial (28 de julio de 1914-11 de noviembre de 1918) nos permite reflexionar sobre un acontecimiento que pese a su importancia está fuera de la memoria colectiva de los mexicanos. Aunque no fuimos ajenos a la guerra y sus consecuencias, los hechos se dieron muy lejos de nuestras fronteras y nuestra propia revolución ha ocultado la trascendencia de la misma para nuestros ojos. ${ }^{1}$ Cuando se revisa la obra multimedia sobre la Primera Guerra Mundial, es notoria la abundancia de textos y documentales referentes a los aspectos militares y político-diplomáticos de la guerra, con diferente grado de detalle y amplitud. En contraste, casi no existen textos ni documentales acerca de los aspectos y consecuencias económicos de la guerra.

\footnotetext{
${ }^{1}$ Como ejemplo estarían la importancia vital que las exportaciones de petróleo mexicano comenzaron a tener para la flota del Reino Unido, la persecución de PanchoVilla por las tropas del general "Black Jack" Pershing - futuro comandante en jefe de las tropas de Estados Unidos en Europa- y el papel del famoso "telegrama Zimmermann" en la intervención de Estados Unidos en la Primera Guerra Mundial; estos hechos aparentemente aislados demuestran la intervención real directa o indirecta de México.
} 
Lo económico es tratado como entorno o condicionante de los acontecimientos militares y políticos o como simples datos aislados dichos de paso.

Este artículo comienza con una extensa y muy necesaria sección de antecedentes. La segunda sección desarrolla las temáticas económicas surgidas por el fracaso de los planes militares: ¿cómo se financió la guerra y las consecuencias inmediatas del financiamiento? y las modificaciones productivas originadas por la guerra. La tercera sección trata sobre el uso de recursos coloniales y el involucramiento de participantes adicionales en el conflicto. Las consecuencias de largo plazo son desarrolladas en la cuarta sección: las consecuencias económicas de la paz de Versalles, especialmente el pago de las reparaciones de guerra, y los cambios productivos y sociales originados. Finalmente desarrollamos las consecuencias económico-sociales a manera de conclusión.

Creo importante continuar con una reflexión de mayor envergadura y profundidad que nos permita averiguar cómo algunas economías de mercado, abiertas e interdependientes solucionaron el esfuerzo de guerra que: a) las privó casi de inmediato de factores productivos, de mercancías y de servicios indispensables en un momento en que incrementar la producción y la productividad eran prioritarios, $b$ ) las obligó a un enorme esfuerzo fiscal porque las necesidades gubernamentales se incrementaron y los recursos escasearon, y c) se alteró el patrón monetario, se dispuso de la riqueza acumulada y se hipotecaron los ahorros futuros, entre otras cosas.

\section{Los antecedentes}

El pretexto oficial para el inicio de la Primera Guerra Mundial fue el asesinato del archiduque Francisco Fernando, heredero de la trono de Austria-Hungría y de su esposa, Sofía Chotek; por el extremista serbio-bosnio Gavrilo Princip el 28 de junio de 1914 en Sarajevo. ${ }^{2}$ Como consecuencia Austria-Hungría envió un ultimátum a Serbia el 23 de julio; Serbia no cumplió con las condiciones exigidas que implicaban su desaparición como estado independiente y Austria-Hungría movilizó sus tropas y declaró la guerra el 28 de julio.

Como consecuencia de las alianzas existentes, desarrolladas durante la llamada "belle époque" o "la paz armada" (1871-1914), debidas principalmente a las rivalidades imperialistas; un conflicto local se generalizó y se convirtió

\footnotetext{
2Aunque el atentado fue ejecutado por la "Joven Bosnia" fue organizado y financiado por "la Mano Negra" organización nacionalista relacionada con el ejército serbio, dirigida por el oficial Dragutin Dimitrievic.
} 
en una guerra mundial, dado el carácter global de las potencias que intervinieron. Las alianzas políticas existentes configuraban dos bandos: los aliados Rusia, protectora de Serbia, y Francia por una parte, contra los llamados imperios centrales (Drei Bund); Alemania, Austria-Hungría e Italia. Sin embargo, el estallido de la guerra y la aplicación del plan Schlieffen por Alemania modificaron la lista de contendientes. Italia abandonó la alianza, declarándose neutral, pretextando el carácter defensivo y no ofensivo del pacto. ${ }^{3}$ Por su parte, el Reino Unido ${ }^{4}$ participó al considerarse garante de la neutralidad e independencia de Bélgica invadida por Alemania el 4 de agosto de $1914 .^{5}$

Debido a la política de alianzas y a los incidentes previos de la guerra, entre los que destacan; la segunda guerra de los Boers 1899-1902, los incidentes de Marruecos 1904-1906 y 1911, las guerras Balcánicas, primera 1912 y segunda 1913. Las potencias imperialistas habían comenzado una carrera armamentista que ya se reflejaba en las economías y las finanzas públicas previas a la guerra. De acuerdo con los datos manejados por Hobsbawn (2013), la tasa media de crecimiento del gasto en armamento pasó de $1.81 \%$ en la década de 1880 , a 2.69 en la de 1890, a 3.46 en la de 1900 (véase Cuadro 1).

Desglosando la competencia militar y económica en dos aspectos; el naval y el militar, tendremos una buena ilustración de la situación existente en el verano de 1914. 1) La rivalidad marítima entre el Reino Unido royal navy y el Segundo Reich Alemán (1871-1918) marina imperial alemana, Kaiserlische Marine, Hochseeflotte se acentúa en los años previos a la guerra. El despegue económico, naval y militar de Alemania en el último tercio del siglo XIX y principios del siglo xx, amenazó las colonias, los mercados y el dominio mundial inglés que garantizaba "la Entente Cordiale". La marina de guerra alemana fue usada como instrumento fundamental para el desarrollo de un

\footnotetext{
3 En realidad el primer ministro italiano Antonio Salandra (1853-1931) invocó el "sacro egoísmo", es decir, que Italia participaría en el bando que le ofreciera más territorios.

${ }^{4}$ En la actualidad, se debate si en realidad el Reino Unido intervino para defender la independencia de Bélgica o sólo fue un pretexto para participar en la guerra y evitar una posible derrota francesa que fortalecería demasiado la posición de Alemania y amenazaría los intereses del Reino Unido en el largo plazo. Boardman, Terry "Belgian Neutrality and the British Decision for War" y "Britain's Responsability in 1914 for wwl" y Royal Geographical Society "Britain Should Not have Fought in WwI"

${ }^{5}$ Parece ser que el Reino Unido prefirió continuar la política de Entente Cordiale o entendimiento cordial, que consistía en una política de aproximación entre Francia e Inglaterra después de siglos de beligerancia. Esta política inició bajo los gobiernos de los reyes Victoria de Inglaterra y Luis Felipe de Orleans en 1845; continuó entre los diferentes gobiernos de la "Inglaterra victoriana" (18371901) y "eduardiana"(1901-1910) con los distintos regímenes de Francia; la presidencia (18481852) y el imperio de Luis Napoleón III (1852-1870) y posteriormente con la $3^{a}$ república francesa (1870-1940), marcando un entendimiento y cooperación en la expansión colonial.
} 
imperio colonial alemán, pasando de ser la cuarta de Europa a ser la segunda; rebasando a Francia en mar y en tierra, amenazando la supremacía naval del Reino Unido de acuerdo a los datos de Hobsbawn (2013) (véase Cuadro 2).

Se considera al almirante Alfred von Tirpitz (1849-1930) como el creador de la marina de guerra e inspirador de la doctrina Teoría del Riesgo de acuerdo con la cual; la marina de guerra alemana sería equivalente a 2/3 de la flota inglesa, pero con superioridad en equipo y entrenamiento, capaz de imponer su concentración de poder en el mar del norte frente al poderío global, supuestamente disperso, de la royal navy. La contraparte inglesa, el almirante John A. Fisher primer lord de almirantazgo a partir de 1904, retoma la política tradicional inglesa: la royal navy, como la primera fuerza naval del mundo debe superar a la suma de 2 a. y 3 a. armadas del mundo. Con Fisher, Inglaterra comenzó un proceso de renovación naval que alcanzó una nueva dimensión estratégica, tecnológica y económica con la botadura del acorazado Dreadnought "sin miedo a nada "en 1906, que volvió obsoletos a los acorazados anteriores, especialmente a una tercera parte la marina de guerra alemana, reiniciando la carrera naval previa a la Primera Guerra Mundial. ${ }^{6}$

En lo estratégico; los ingleses aprovecharon la derrota rusa en la guerra ruso-japonesa de $1905,{ }^{7}$ pactando con los rusos y renovaron la Entente Cordiale con Francia. Así el R.U. "cedió” el mediterráneo a los franceses, concentrando sus mejores unidades en el mar del norte, para un posible enfrentamiento con Alemania. La nueva doctrina estratégica se basó en bloquear a Alemania y no en la búsqueda de victorias navales definitivas. Pero, enfaticemos que la guerra comenzó antes de que la marina imperial alemana tuviera el potencial de desafiar a la royal navy.

Mención aparte merece la competencia de las marinas mercantes de Alemania y el Reino Unido por el dominio del transporte de carga y pasajeros en diversas partes del mundo; el más lucrativo entre Europa y Estados Unidos; seguido por las rutas coloniales y las relacionadas con países no coloniales. Entre 1881 y 1910, la flota mercante alemana pasó del cuarto al tercer lugar;

\footnotetext{
${ }^{6}$ Los acorazados de la clase "sin miedo a nada" innovaron la doctrina de construcción de acorazados: 1) unificaron e incrementaron el número de cañones navales de primera línea de 4 a 10 cañones, de calibre $305 \mathrm{~mm}(12$ ”), con una segunda línea 27 cañones de calibre $76.2 \mathrm{~mm}$. Los acorazados tenían propulsión basada en 4 motores de vapor con 4 hélices, alcanzando los 21 nudos de velocidad máxima. Estos nuevos modelos obligaron a las potencias rivales a una carrera por su construcción y posesión. Posteriormente, la incorporación de los motores diesel obligó a que las potencias navales buscaran en adelante tener fuentes de producción y abastecimiento seguro de petróleo y no de carbón.

${ }^{7}$ Rusia perdió dos de sus tres flotas en la guerra ruso-japonesa; la del Pacifico en Shantung el 10 de agosto de 1904 y la del Báltico en la batalla de Tsushima mayo 27-28 de 1905.
} 
sólo después del Reino Unido y Estados Unidos, pero en términos relativos casi duplicó su participación en el total mundial (véase Cuadro 4). La disputa por el listón azul, para el viaje más rápido entre Europa y Estados Unidos, involucró en algún momento a las líneas inglesas Cunard y White Star contra las líneas alemanas Hamburg-Amerika (Hapag) y Norddeutscher Lloyd. ${ }^{8}$

Por lo que respecta a los preparativos y gastos militares previos a la Primera Guerra Mundial. La evolución económica y demográfica fue favorable a Alemania sobre Francia. ${ }^{9}$ Por consecuencia Francia se sintió obligada a adoptar mediadas extremas internas y externas, que elevaran el número de efectivos a disposición del ejército francés (conscripción obligatoria y extendida) y que aseguraran aliados con ejércitos numerosos, para enfrentar a Alemania y Austria-Hungría, ${ }^{10}$ la elección natural recayó en Rusia. Esta disparidad se puede apreciar en el Cuadro 3, tomado de Hobsbawn (2013).

Si bien la construcción del imperio alemán por el canciller Otto von Bismarck $^{11}$ (1815-1898) alrededor del reino de Prusia y la dinastía Hohenzollern ${ }^{12}$ se dio a través de 3 guerras. ${ }^{13}$ Una vez logrados sus objetivos, Bismarck

\footnotetext{
${ }^{8}$ Debe de aclararse que en su apogeo cada línea, alemana o inglesa, tenía un diseño propio de buque (tipo o clase), buques insignia, nombres característicos para sus navíos, rutas propias y segmentos de mercado preferidos, por ejemplo: la White Star poseía el RMS Titanic, (hundido en1912), el RMs Britannic (hundido en 1916) de la clase Olympic. La Cunard posé́a los RMs Queen Mary, Rms Queen Victoria, y al Lusitania (hundido en1915) etc. Por su parte, Hapag poseía el Amerika y elYpiranga entre muchos otros.

${ }^{9}$ Hjalmar Schacht (1956), aporta datos en sus Confessions., capítulo xv, sobre el desarrollo económico alemán: para el período 1870-1910, se destaca el incremento de la población alemana que creció en un 52\% mientras que la de Reino Unido 37\% y la de Francia sólo 8 por ciento.

${ }^{10}$ Después de la guerra franco-prusiana 1870-1871, Francia desarrolló una política que contemplaba a Alemania como principal amenaza. Francia buscó alianzas que contrarrestaran esa amenaza y permitieran una revancha; pero la población francesa de "la belle époque" y su tasa de natalidad la ponían en desventaja. Por ello, el presidente Raymond Poincaré (1860-1934), originario de la Lorena anexada a Alemania y republicano conservador, estableció la ley de 3 años que aumentó el servicio militar de 2 a 3 años. Políticamente, sólo se opuso la sección francesa de la internacional (SFIO) encabezada por Jean Jaurès (1854/1914), asesinado el 31 de julio de 1914. La Ley de 3 años, en teoría, aumentaba los efectivos del ejército francés de 480 mil a 750 mil soldados; estimando un ejército alemán de 850 mil efectivos. En la política exterior esta necesidad práctica llevó a Francia a una política de reconciliación con Rusia, después de la guerra de Crimea (1853-1856), que contaba con ejércitos estimados en varios millones de hombres y a proseguir con la Entente Cordiale con el Reino Unido.

${ }^{11}$ En términos militares las guerras de Bismarck fueron dirigidas por Helmuth von Molke, o "Molke el viejo" (1800-1891).

${ }^{12}$ Los emperadores de la dinastía Hohenzollern o káiser de Alemania fueron: Guillermo I, Federico III y Guillermo II 1871-1918.

${ }^{13}$ Las guerras de Bismarck son las siguientes: la primera guerra contra Dinamarca en 1864, Prusia obtuvo Lauenburg y Schleswig-Holstein, la segunda guerra contra Austria- Hungría en 1864, Prusia obtuvo Hesse, Fráncfort, Hannover y Nassau, la tercera guerra contra Francia en 1870 Prusia obtuvo Alsacia y Lorena; proclamando el II Reich alemán, en Versalles 18 de enero de 1871, por medio de la unificación del reino de Baviera; el reino de Sajonia, el reino de Wurtemberg, el ducado de Baden
} 
no siguió una política de guerra abierta sino de búsqueda de equilibrios en los escenarios europeo y mundial. Alemania se reconcilió con Austria-Hungría y la convirtió en su aliada; trató de aislar a Francia y de asegurar la neutralidad del Reino Unido y de Rusia. El abandono de la política exterior de Bismarck ${ }^{14}$ por el káiser Guillermo II, permitió el acercamiento de Francia a Rusia e Inglaterra, con consecuencias lamentables para Alemania. ${ }^{15}$

\section{El fracaso de los planes militares y el alargamiento de la guerra ${ }^{16}$}

\section{La alianza entre Francia y Rusia de 1890 provocó un gran temor en Alemania porque representaban el riesgo de pelear en dos frentes; al este con Francia y oeste con Rusia. El conde Alfred von Schliffen (1833-1913), jefe del estado mayor, desarrolló el plan Schlieffen, ${ }^{17}$ que trataba de dar un primer golpe a}

y la confederación alemana del norte (Norddeutscher Bund 1867-1871).

${ }^{14}$ La política de Bismarck después de la guerra consistió en aislar a Francia; en 1873 con "el Tratado de los 3 emperadores"; Austria-Hungría, Rusia y Alemania acordaron sus diferencias. En 1887 con "el Tratado de reaseguramiento" Bismarck buscó la neutralidad rusa en caso de guerra entre Francia y Alemania; a cambio de la neutralidad de Alemania en una guerra Rusia contra Austria; además de negociar sus intereses en los Balcanes, el Bósforo y los Dardanelos (Turquía).En 1890, con la renuncia de Bismarck, el káiser Guillermo II se negó continuar la política anterior y Alemania no renovó "el Tratado de los 3 emperadores". El acercamiento de Alemania a Austria-Hungría, condujo a que Rusia buscara aliarse a la república francesa y la neutralidad del R.U.

${ }^{15}$ Debido a la fuerte expansión de Alemania en todos los terrenos; el Reino Unido decidió salir del "splendide isolement" (esplendido aislamiento) llevado adelante por el primer ministro Benjamín Disraelí (1804-1881), retomando la Entente Cordiale en 1904 con Francia y reconciliándose con Rusia, a través de las conferencias anglo-rusas del 31 de agosto de 1907, por medio de las cuales acordaron sus diferencias respecto a Afganistán, Persia y Tíbet.

${ }^{16}$ La Primera Guerra Mundial o la "Gran Guerra", comenzó como otra guerra europea, se pensaba por las experiencias previas que duraría unas semanas o en el peor de los casos meses y tendría efectos limitados. Sin embargo, pese a su impacto mundial y la lucha en los múltiples frentes, fue en el frente occidental dónde se involucraron las potencias militares más poderosas, los recursos militares y económicos más grandes y las innovaciones tecnológicas. Por ello al analizarla, se privilegia el frente occidental donde predomina la "guerra de posiciones o trincheras" sobre el frente oriental y otros, donde predomina la "guerra de movimientos".

${ }^{17}$ El conde von Schlieffen ideó el plan de guerra alemán que suponía que Austria-Hungría detendría a Rusia en el frente del este con una ayuda simbólica de Alemania, 5\% del ejército alemán: El fracaso de tres ofensivas austro-húngaras sobre Serbia en 1914 obligaron a retirar tropas austrohúngaras del frente ruso y a enviar más tropas alemanas, llegando a 15\% del total de los efectivos alemanes. El plan también preveía el ataque francés (plan XVII) por Alsacia y Lorena, proponiendo una guerra defensiva con sólo 5\% de la tropa; Helmuth von Molke "el joven" incremento esa cifra a 15\%, Así en vez de atacar a Francia con $90 \%$ del ejército alemán (Schlieffen), Molke sólo dispuso de $60 \%$ enfrentando dos dificultades adicionales: una resistencia no esperada de Bélgica y la participación del Reino Unido, supuestamente neutral, con una fuerza expedicionaria de 100 mil efectivos. El plan Schlieffen y la carrera militar de von Molke fracasaron en la batalla del Marne durante septiembre de 1914. 
Francia; a través de Bélgica y Luxemburgo para después atacar a Rusia. El plan fue reformado por Helmuth von Molke "el joven" (1848-1916), sobrino de Molke "el viejo". Como hemos mencionado todos los países que intervinieron inicialmente en la guerra tenían planes militares previamente estudiados y aprobados que fueron seguidos casi al pie de la letra, confinado en que ello condujera a una guerra corta y una victoria militar inmediata. En la práctica los planes militares de todos los países fracasaron.

Al fracasar el plan Schlieffen con la batalla del Marne, siguió un breve periodo conocido como "la carrera al mar" que termino dibujando un frente lleno de trincheras que se prolongaba de la frontera de Suiza al mar del norte. En la guerra de trincheras, el objetivo fundamental consistió en la derrota del enemigo por agotamiento económico y apabullamiento material más que por batallas terrestres o navales frontales. Para fines de 1914, los ejércitos profesionales previamente existentes estaban destruidos, mientras que la guerra se prolongaba más allá de lo calculado por los planes estratégicos.

\section{El costo de la guerra ${ }^{18}$}

En todos y cada uno de los países surgió la necesidad de prolongar el esfuerzo de guerra. Ninguno de los contendientes tenía "un plan B" seriamente estudiado que contuviera apartados económicos sobre cómo llevar adelante una economía de guerra. Las necesidades que surgieron se fueron solucionando sobre la marcha. Primero, la reconstrucción de los ejércitos para la campaña de $1915 .{ }^{19}$ Es decir, 1) se inició el reclutamiento obligatorio ${ }^{20}$ de los jóvenes en "edad militar" que se mantuvo mientras duro el conflicto. 2) se produjeron modificaciones en la esfera productiva y en las finanzas públicas para alimentar, equipar, entrenar, transportar, dar atención médica y sanitaria a las tropas. ${ }^{21}$ 3) la necesidad de sustituir a la mano de obra "ausente" llevó a

\footnotetext{
${ }^{18}$ No nos referimos a los estimados finales sobre el terrible costo total de la guerra en términos humanos y materiales sino al financiamiento de los acontecimientos inmediatos.

${ }^{19}$ Los testimonios artísticos sobre esta primera generación son abundantes, basta citar algunas de las novelas clásicas sobre el tema: de Erich María Remarque (1898-1970), Sin novedad en el frente 1929 y sus subsiguientes películas 1930 y 1979; Tempestades de acero 1920 de Ernst Jünger (1895-1998), por el lado alemán; La mano cortada 1946 del suizo Blaise Cendrars (1887-1961), en el lado francés, Adiós a todo eso 1929 del inglés Robert Graves (1895-1985), y “Adiós a las armas” 1929 de E. Hemingway (1899-1961), por Estados Unidos.

${ }^{20}$ En el Reino Unido no se recurrió inmediatamente al reclutamiento obligatorio sino se hizo un llamado al enrolamiento voluntario, el llamado "ejército de lord Kitchener" en 1915. Sin embargo, en el verano de 1916 se aprobó la conscripción obligatoria y universal para hombres entre 18 y 41 años.

${ }^{21}$ Se dio un gran impulso a la intervención económica del estado y a las políticas de planeación de la producción alejando a la economía y al estado de las concepciones liberales de ambos.
} 
la incorporación de grandes contingentes de mano de obra femenina ${ }^{22}$ tanto para la producción militar como no militar. 4) aunque el aspecto naval tomó gran relevancia, las operaciones navales no se llevaron de acuerdo a la teoría tradicional. ${ }^{23}$

De acuerdo con Schacht, en Alemania la normalidad económica continuó durante los primeros meses de la guerra. ${ }^{24}$ Conforme la guerra se acercó al año los problemas fueron emergiendo con la escasez de materias primas y alimentos, y sus efectos inflacionarios. Los controles y el racionamiento de materiales estratégicos fueron apareciendo mientras los productos desaparecían del mercado: oro, divisas, metales, azúcar, cacho etc. Posteriormente, frente a la escasez de recursos propios, los alemanes trataron de explotar los territorios ocupados en su beneficio. Se propuso en las zonas ocupadas un retorno a la "normalidad" evitando la "requisa militar" obligatoria, pagando a cambio el costo del mantenimiento del ejército de ocupación y contribuyendo al esfuerzo bélico alemán. Esta técnica de explotación fue corregida y aumentada brutalmente durante la Segunda Guerra Mundial. ${ }^{25}$

Schacht argumenta que uno de los más grandes errores de Alemania durante la guerra consistió en una errónea política fiscal para el financiamiento del esfuerzo bélico, la venta excesiva de "prestamos de guerra" por parte del gobierno, redujo los activos de los ahorristas alemanes a papel sin valor al termino de la guerra. Schacht consideraba el financiamiento británico de la guerra, a través de impuestos, especialmente sobre las ganancias extraordina-

${ }^{22}$ La participación de la mano de obra femenina provocó a la larga una gran transformación social: durante la guerra las mujeres salieron de sus hogares, sobre ellas recayó el esfuerzo productivo y obtuvieron ingresos e independencia económica y social. En la posguerra, la generalización del sufragio universal y algunas reivindicaciones de género obtuvieron más apoyo y fuerza que en los días anteriores a la guerra.

${ }^{23}$ En esta guerra el enfrentamiento naval tradicional tuvo contadas acciones. Las batallas de Coronel (1- XII-1914) y las Malvinas (8-XII-1914), al principio de la guerra y la batalla de Jutlandia (31 de mayo y 1 de junio de 1916) a mediados de la misma. La estrategia naval seguida fue de desgaste económico y material, la flota de superficie británica predomino al bloquear los puertos alemanes, mientras la flota alemana actuó a la defensiva. La ofensiva y bloqueo alemán de los puertos ingleses se dio a través de la guerra submarina.

${ }^{24}$ Schacht, H. (1956), Confessions. capítulo xvi.

${ }^{25}$ Sin olvidar que en gran parte los genocidios y otras brutalidades atribuidos al 3er Reich están vinculados al esfuerzo de guerra. Parece ser que los ministros de armamento y guerra del 3er Reich; Fritz Todt (1891-1942) y Albert Speer (1905-1981), en gran parte responsables de estos crímenes, cuestionaron la eficiencia pero no la moralidad, del esfuerzo de guerra alemán durante la Segunda Guerra Mundial. Aunque la maquinaria industrial militar alemana se mantuvo más allá de lo esperado, el uso de mano de obra extranjera "semi-esclava, no confiable y de baja calidad" fue políticamente rentable porque evitó la movilización de mano de obra femenina y masculina alemana; pero económica y militarmente ineficiente, reduciendo la eficiencia, la calidad y el potencial productivo alemán. 
rias, mucho mejor y más justo desde el punto de vista social. De esta forma, el financiamiento alemán de la guerra produjo escasez y mercado negro en un primer momento, especulación e inflación en el largo plazo. ${ }^{26}$

J. M. Keynes reexaminó el problema del financiamiento de la Primera Guerra con más detenimiento durante los primeros meses de la Segunda Guerra. Entre septiembre de 1939 y febrero de 1940, Keynes reflexiona y escribe un pequeño libro cómo pagar la guerra. ${ }^{27}$ En él incorporó sus experiencias de la Primera Guerra Mundial a los nuevos cuestionamientos y realidades.

Keynes insiste en que la economía pasó durante 1939 del desempleo al pleno empleo sin que esto significara un incremento del consumo; porque la economía de guerra imponía algunas restricciones. 1. el país tiene que reorganizar su producción para elevarla a un máximo permitido por sus recursos y nivel organizativo. 2. de la suma de la producción y las importaciones se deben deducir las exportaciones y las necesidades de la guerra; lo que queda debe dedicarse al consumo civil. 3. se debe llegar a un compromiso entre las necesidades militares y civiles. 4. lo ideal sería el financiamiento de la guerra a través de los impuestos a $100 \%$ pero eso no es posible, en algún momento se tiene que recurrir al crédito. 5. también sería ideal no llegar al control total de la producción y permitir el libre juego de las fuerzas del mercado, una vez que las necesidades militares y las necesidades mínimas de consumo civil sean satisfechas. 6. no se puede permitir que la escasez de un producto quede en manos del dinero disponible por el público y de su propensión a gastarlo. Finalmente Keynes propone 7. transferir parte del costo de la guerra a las colonias.

Dado que hay pleno empleo, con mayores ingresos y menor disposición de bienes y servicios para satisfacer las necesidades de consumo, se propone una política impositiva redistributiva y una política de endeudamiento que reduzca el dinero en la economía y las presiones inflacionarias. Esta política razonable debe permitir un máximo esfuerzo de guerra sin al agotamiento de las reservas propias ni del crédito externo.

\footnotetext{
${ }^{26}$ Schacht, H. (1956), Confessions... capítulo xviI.

${ }^{27}$ J. M. Keynes (2009)," cómo pagar la guerra" publicado como capítulo en el libro titulado Ensayos de persuasión.
} 


\section{Mundialización de la guerra: el uso de los ejércitos y las guerras coloniales}

Es evidente que los franceses e ingleses recurrieron en mayor medida que los alemanes y austro-húngaros a los recursos y a los ejércitos coloniales para el mantenimiento de su esfuerzo de guerra (véase Cuadro 5). ${ }^{28}$ Para los imperios centrales, el uso de esas tropas coloniales aliadas era "juego sucio", sobre todo en los escenarios más difíciles donde las tropas hindús, gurkas, africanas, magrebíes e indochinas fueron usadas como tropas de choque o carne de cañón. ${ }^{29}$

Las recursos y especialmente las tropas de los dominios ingleses: Canadá, Australia y Nueva Zelanda, fueron ocupadas generalmente en frentes secundarios, Gallipoli y en casos de emergencia: Flandes octubre de 1914. Sin embargo, esto no significo un menor esfuerzo económico para los dominios ni mayor seguridad para sus tropas. El fracaso de operación de Gallipoli (1915) contra el imperio turco represento una gran sangría para los dominios de Oceanía, y fue un punto de quiebre en su historia y una referencia cultural de primer orden. ${ }^{30}$ En la guerra colonial africana, no hubo operaciones de mayor importancia, excepto la resistencia triunfal en, la entonces llamada Rodesia del norte, del Coronel Paul von Lettow-Vorbeck, ascendido a general en el último edicto del káiser y recibido con honores militares de vencedor en la "Puerta de Brandenburgo" en marzo de 1919.

\section{El juego de suma y resta ${ }^{31}$}

Una vez estabilizada la guerra de trincheras, cada bando trató de ganar aliados para su causa y reducir el número de enemigos. No se dejó de lado ningún recuso para lograrlo, usando a los países como piezas de ajedrez: abundaron las

\footnotetext{
${ }^{28}$ Se calcula que del total de soldados del R.U. que intervinieron en la guerra: 1) 700 mil, 9\% del total pertenecían al viejo ejército colonial, 2) se reclutaron 4 millones de ingleses, $48.9 \%$, del total, del resto del R.U. (Escocia, Gales e Irlanda) se obtuvieron 970 mil, 11\%, de los dominios procedían otros 965 mil, 11\% (Canadá, Australia, Nueva Zelanda y Sudáfrica principalmente), las tropas hindús y de color alcanzaron los 1 millón 530 mil efectivos $18.6 \%$ del total. Por su parte, Francia obtuvo 600 mil soldados africanos e indochinos.

${ }^{29}$ El imperio austro-húngaro trato de no emplear tropas eslavas contra Rusia y Serbia, pero recurrió al uso masivo de las mismas en el frente italiano. En el imperio de los Habsburgo, los austriacos (12 millones) y húngaros (10 millones) representaban una mayoría apretada sobre los 15 millones habitantes de otras nacionalidades: 1. polacos, 2. checos, 3. eslovacos, 4.eslovenios, 5. croatas, 6.bosnios, 7. rumanos, 8. serbios y 9. italianos

${ }^{30}$ Para referencias cinematográficas al respecto: «Gallipoli»(1981), película australiana de Peter Weir con Mel Gibson. La versión turca es contada por el film "Canakkale 1915” de 2012.

${ }^{31}$ Con datos de British Army Statistics y Spencer Tucker (2005).
} 
promesas sobre reivindicaciones territoriales; el oportunismo, los sobornos, el espionaje, los golpes de estado y la ocupación de países neutrales por ambas partes. En agosto de 1914, Japón se apoderó de las colonias alemanas en Asia con la aprobación aliada y la oposición política de los Estados Unidos, sin mayores compromisos para los demás frentes.

Para debilitar y desviar al ejército ruso de las fronteras alemanas y austriacas; el eje se agenció la alianza de Turquía, octubre de 1914; rusos y turcos se enfrentan en el Cáucaso. ${ }^{32}$ La respuesta inglesa consistió en ataques sobre Palestina desde Egipto (1 millón 200 mil efectivos totales) y sobre Mesopotamia desde la India (900 mil efectivos). Sin embargo, la lucha más enconada en este frente se producirá entre las tribus árabes armadas por el Reino Unido y Turquía. ${ }^{33}$ El 25 de abril de 1915 el Reino Unido y Francia atacaron directamente la capital turca a través de Gallipoli. El número de efectivos usados en toda la operación alcanzo 470 mil efectivos del R.U. 80 mil franceses y 315 mil turcos.

El 23 de mayo de 1915, Italia entró a la guerra contra sus ex-aliados a cambio de importantes promesas territoriales. Entre 1915 y 1918, ocurrieron 10 campañas bélicas en ese frente. Spencer Tucker estima el uso de 5 millones de efectivos italianos en total con pérdidas cercanas a 700 mil bajas. Bulgaria se incorpora a la guerra en octubre de 1915, Bulgaria, Alemania y AustriaHungría invaden Serbia. El ejército serbio se retira al mar en noviembre, mientras Reino Unido y Francia ocupan Grecia que declara la guerra al eje en junio de 1916, 400 mil efectivos del R.U. participaron en total. Las tropas inglesas, francesas, rusas y griegas combaten a Bulgaria desde octubre de 1916. Se calcula que Bulgaria movilizó 1.2 millones de soldados y tuvo alrededor de 100 mil bajas. Rumania entra en la guerra del lado aliado en agosto de 1916, inmediatamente es atacada por Bulgaria, Alemania y Turquía en septiembre. En diciembre, Rumania está completamente ocupada. Se estima que Rumania movilizo 1 millón 200 mil soldados con pérdidas de 340 mil efectivos.

En 1917 ocurrieron algunos hechos fundamentales: 1) la entrada de Estados Unidos a la guerra, el 2 de abril: la llegada de tropas y equipo se hará realidad meses después; en total los Estados Unidos movilizaron 4 millones 740

\footnotetext{
${ }^{32}$ Se estima que Turquía empleo un total de 7 millones de efectivos militares con pérdidas cercanas a 850 mil bajas. Sin embargo, el aspecto más lamentable de la intervención de Turquía consistió en el genocidio de los armenios y asirios (al menos un millón de víctimas), con el pretexto de su participación pro-rusa en el conflicto.

${ }^{33}$ Los ingleses usaron al famoso Lawrence de Arabia (T. E. Lawrece 1888-1935) para comprar el favor de los árabes con promesas de independencia mientras los alemanes por medio de Wilhelm Wassmuss y Max von Oppenheim trataron de desarrollar una "jihad” antibritánica.
} 
mil efectivos y tuvieron 210 mil bajas. 2) la retirada rusa de la guerra (acuerdos de Brest-Litovsk, diciembre 1917-marzo 1918), fue debida a las constantes derrotas rusas frente a los ejércitos alemanes en el frente oriental y al triunfo de la revolución de octubre; (6-7 de noviembre) de 1917. ${ }^{34}$ La nueva URss cedió los estados bálticos y Polonia a Alemania, mientras comenzaba la guerra civil entre bolcheviques y rusos blancos. 3) el hundimiento del frente italiano en Caporetto, noviembre de 1917, provocó la llegada de refuerzos aliados; 145 mil efectivos del R.U.

En verano de 1918, Alemania trató de ganar la guerra, antes de la llegada del grueso de los ejércitos de los Estados Unidos, lanzando importantes ofensivas en el frente occidental en agosto (Kaiserschlacht). Sin embargo, el fracaso de esas ofensivas y el contra-ataque aliado en octubre, convencieron a los mariscales alemanes Paul von Hindenburg y Erich Ludendorff de la imposibilidad de la victoria alemana. En el otoño de 1918, los aliados de Alemania se fueron derrumbando uno tras otro: Bulgaria el 31 de septiembre, Turquía el 30 de octubre, Austria-Hungría el 3 de noviembre. Finalmente, Alemania capitula el 11 de noviembre de 1918 a las 11 A.M.

\section{Las consecuencias de largo plazo}

La paz posterior a la primera guerra mundial, oficializada en los Tratados de Versalles con Alemania junio de 1919 y de Saint Germain con Austria septiembre de 1919, se presenta en la reflexión actual, como una salida en falso a las problemáticas ocasionadas por la guerra mundial y la destrucción del orden preexistente. Se considera que estos tratados no solucionaron los verdaderos problemas económicos, políticos y sociales; que sólo sirvieron para que en la siguiente década se instalaran regímenes autoritarios en la mayor parte del continente y a la larga se desencadenara la Segunda Guerra Mundial.

\section{Las reparaciones de guerra (el debate Keynes-Ohlin)}

Las reparaciones de guerra exigidas por los aliados a los perdedores, sumaron cifras gigantescas imposibles de pagar por los países perdedores; éstos estaban físicamente destruidos, económicamente agotados y endeudados, evidentemente no podían hacer frente a los compromisos pactados. El cumplir con estos compromisos implicaba, la consecución de dos objetivos macroeconómicos de gran dificultad: 1) un superávit de las finanzas públicas para

\footnotetext{
${ }^{34} \mathrm{El}$ calendario ruso ortodoxo lleva 12 días de retraso con respecto al calendario occidental.
} 
ser transferido a los vencedores, 2) un superávit de la cuenta corriente de la Balanza de Pagos para convertir el superávit fiscal en divisas.

Bertil Ohlin (1899-1979) argumentó que el ajuste podría darse de forma automática por medio de las libres fuerzas del mercado. J. M. Keynes (1883-1946) consideró que tal ajuste era imposible debido a la incapacidad real de realizar los ajustes macro-económicos necesarios. En los años de la década de 1920, Alemania tuvo que recurrir al doble proceso de devaluación-inflación, deteriorando las condiciones de vida del país, para tratar obtener ambos superávit. Sin embargo, se enfrentó a un incremento del proteccionismo de los propios países beneficiarios del pago de las reparaciones. En la década de 1980, el problema de la "Deuda Externa" de los países en desarrollo, condujo a una revisión del viejo debate.

Después de la Primera Guerra Mundial, J. M. Keynes denunció la destrucción legal, por el Tratado de Versalles, de la economía alemana y de Europa central, al través de varios mecanismos, entre los que destacan: 1. la destrucción de la flota mercante alemana, usando sus barcos y activos para el pago de reparaciones de guerra. 2. la destrucción del poderío industrial alemán privándolo de los yacimientos de carbón (Alsacia) y acero (el Sarre), bases de la industria del siglo XIX y principios del Xx. ${ }^{35} 3$. la destrucción de eslabonamientos industriales entre Alemania y sus vecinos europeos.

\section{Las transformaciones productivas ocasionadas por la guerra}

La Primera Guerra Mundial fue la primera guerra plenamente tecnológica; las guerras de Crimea, civil de los Estados Unidos (1861-1865) y franco-prusiana, sólo representaron ensayos parciales del mismo fenómeno, que enunciaban como la capacidad productiva y científico técnica de fines del siglo xIX y principios del xx, terminó destruyendo el viejo arte de la guerra occidental. Las estrategias, las tácticas y las operaciones militares concretas se vieron completamente condicionadas por la capacidad productiva/destructiva de la ciencia y la tecnología.

\footnotetext{
${ }^{35}$ En el Tratado de Versalles se obligó a los alemanes a:1) ceder parte de su flota de guerra a los ingleses; en la realidad la tripulación alemana prefirió hundir su propia flota antes de ser entregada en la base naval de Scapa Flow, islas Orcadas. 2) entregar todos los buques mercantes mayores a 1600 toneladas, la mitad de los buques entre 1600 y 1200 toneladas, y una cuarta parte de los menores a 1000 toneladas. Además del compromiso de construir barcos por 200 mil toneladas para pagar las reparaciones.Véase J. M. Keynes (1987) Las consecuencias económicas de la paz, mención aparte, merece la cesión de la flota de dirigibles alemanes a los aliados terminada la guerra y la construcción por Alemania del LZ 126 rebautizado "USS Los Ángeles" para Estados Unidos.
} 
Las máquinas intervinieron de forma absoluta y definitiva en la guerra; las viejas formaciones napoleónicas y las cargas de caballería son impracticables dado el poder de fuego de la artillería y la fusilería. Ya no se ve al enemigo, la muerte se vuelve impersonal y tecnológica, especialmente con el uso de los gases. ${ }^{36}$ Se abandona la vieja caballería y se le sustituye por maquinas impulsadas por motores de combustión interna; automóviles, tanques, submarinos y aviones. Las batallas navales dejan de depender por completo de la fuerza del viento, las flotas se alejan y el combate naval vive las últimas batallas del viejo estilo; con dos flotas enfrentadas en línea.

Surgieron dos frentes de batalla nuevos; la guerra submarina y la guerra aérea que evolucionan rápidamente desde sus orígenes modestos y primitivos. En la actualidad, ambas han modificado la táctica y la estrategia de la guerra, convirtiéndose en ramas militares definitorias de la guerra del siglo XXI. Los avances científicos y tecnológicos provocados y alentados por la guerra, pronto fueron aplicados y generalizados en el campo productivo civil, conduciendo una gran transformación de las formas de producción. Nuevos productos y servicios disponibles aparecieron en las economías desarrolladas, se generalizaron de los nuevos medios de transporte, y especialmente se consolidó la irrupción del motor de combustión interna como "el motor" del producto rey del siglo xx: el automóvil. Todos y cada uno de estos acontecimientos que afectaron la vida cotidiana mundial durante el resto del siglo.

\section{Conclusión: los cambios económico-sociales resultantes de la guerra}

La desaparición de los viejos de los imperios europeos: 1. Austria-Hungría, 2. Rusia, 3. Turquía, 4. Alemania dio lugar al surgimiento de la Europa de las nacionalidades y promovió el surgimiento de soluciones no democráticas tanto en los nuevas naciones como en los países remanentes de los viejos imperios. El pago de las reparaciones de guerra por Alemania deterioró condiciones de vida del pueblo alemán abonando el camino del nazismo y fomentando el revanchismo que conduciría a la Segunda Guerra Mundial. En Italia el deterioro de las condiciones de vida y la "amenaza" socialista en la posguerra, condujeron al triunfo del Fascismo con el apoyo de la burguesía y la oligarquía terrateniente.

\footnotetext{
${ }^{36}$ Véase el film "Clara Immerwahr" donde se exponen las dificultades personales y matrimoniales entre Clara Immmerwahr y Fritz Haber, ocasionadas por el desarrollo de las armas químicas en el instituto dirigido por Haber.
} 
La revolución rusa permitió el surgimiento de la URSS que encarnó las esperanzas en la utopía socialista, desafortunadamente culminó con en la dictadura estalinista. La modernización de la economía rusa y su transformación en economía soviética se dio con grandes costos económicos, sociales y políticos. El cambio del orden económico y político internacional, se tradujo en el fin del siglo británico y la emergencia de Estados Unidos como la principal potencia, económica, política y militar del mundo.

El esfuerzo de guerra y las tensiones económicas, políticas y sociales que produjo llevo a una nueva redefinición del papel del Estado en la economía; abandonándose el Estado liberal por el pacto "keynesiano" durante la segunda posguerra. La movilización de las mujeres europeas en la administración; la producción, los servicios públicos y sociales, ocasionalmente en el frente de guerra; condujeron al fin de la sociedad "victoriana" con sus valores económicos, sociales y políticos, impulsando el trabajo femenino fuera de la esfera doméstica, el sufragio universal femenino, y la revisión del universo femenino en todos los aspectos; desde la moral pública y privada hasta la moda y los patrones de consumo.

\section{Bibliografía y otros medios consultados}

\section{Publicaciones académicas}

Hobsbawn, Eric (2013), La era del imperio 1875-1914, Editorial Crítica, Barcelona. Keynes, J.M. (2009), Ensayos de persuasión, Editorial Síntesis Madrid.

Keynes, J.M. (1987[1919]), Las consecuencias económicas de la paz, Editorial Crítica, Barcelona.

Schacht, Hjalmar (1956), Confessions of "The Old Wizard", The Riverside Press, Cambridge, Boston.

Tucker, Spencer (2005), The Enciclopedia of World War I. A political, social and military History, Editorial ABC-Clío.

Westwell, Ian (2003), Primera Guerra Mundial, día a día, Editorial LIBSA, Madrid.

\section{Novelas}

Cendrars, Blaise (1980 [1946]), La mano cortada, Editorial Argos-Vergara, Madrid. Graves, Robert (2009 [1929]), Adiós a todo eso, Editorial Universidad Veracruzana, Xalapa. Jünger, Ernst (1987 [1926]), Tempestades de Acero, Editorial Tusquets, Barcelona. 
Economía Informa núm. 392 mayo - junio • 2015

Radiguet, Raymond (2000[1923]), El diablo en el cuerpo, Editorial Pre-textos.

Remarque, Erich Maria (2000[1929]), Sin novedad en el frente, Editorial Porrúa, México.

\section{Películas}

“Cannakale (Gallipoli 1915)” (film Turquía, 2012), Dir. Yesim Sezgin; Baran Akbulut, Ózgür Akdemir. Http// youtu.be/ VA1Ny_pGNVc

"Clara Immerwahr" (Ferbsehfilm Deutschland, 2014), Dir. Harald Sicheritz; Katharina Schüttler, Maximilian Brückner. Http// youtu.be/ GUYxB010Xms

"Gallipoli” (film Australia, 1981), Dir. Peter Weir. Mel Gibson, Mark Lee. Http// youtu.be/ojFxIULdEQo

"JoyeuxNöel /La tregua de navidad" (Film FR/UK/GER, 2006), Dir. Christian Carion, Benno Fürman, Guillaume Canet, Diane Kruger. Http// youtu.be/ IiJUs63qJPE

"Sin novedad en el Frente" (Film E.U.A. 1930), Dir. Lewis Milestone; Louis Wolheim, Lew Ayres.

"Sin novedad en el Frente"(Film E.U.A/R.U. 1979), Dir. Delbert Mann; Richard Thomas, Ernest Borgnine. Http// youtu.be/IQPcOgYsYn8.

"The Trench / La trinchera" (film R.U./FRA, 1999), Dir. William Boyd; Paul Nicholls, James d'Arcy, Daniel Craig. Http// youtu.be/ -8sPo1h3_NO

"The Red Baron / Der rote Baron" (Film Ger, 2008), Dir. Nikolai Müllerschön; Matthias Schweighoffer, Lena Heady. Http// youtu.be/KehpMWk3MSY

"War horse / Caballo de Guerra" (film R.U/E.U.A, 2011), Dir. Steven Spielberg; Jeremy Irvine, David Thewlis.

\section{Documentales}

Die Deutschen 09 "Bismarck und das Deustche Reich" (2010), Stefan Brauburger, Georg Graffe. ZDF Produktion. Deutsch/HD [DOKU] Http// youtu.be/ eiMTB4S2UMc

Die Deutschen 10 "Wilhelm und die Welt" (2010), Stefan Brauburger, Georg Graffe. ZDF Produktion. (Deutsch/HD)[DOKU] Http// youtu.be/ eiMTB4S2UMc

La Grande Guerra. La Storia Viva (2002), Simona Gardini et al. Olimpia Cinematografica.

"Le origini del Conflitto" ep. 1. Http// youtu.be/ IcUmsWiFlwSs.

"Della mobilizazione alla Guerra in Trincea" ep. 2 Http// youtu.be/ IqCRguStOM10. 
"L’Italia in guerra. Izonzo, Fiume de Sangue" ep. 3, Http// youtu.be/ NcODijo388w

“Adomello. La guerra a Quota 3000” ep. 4 Http// youtu.be/ Y0yuzRN-pV0

«Il Fronte Orientale dopoTannenberg» ep 5. Http// youtu.be/ XF5D2av0G24

"Sorizia, Trieste e l'operazione K” ep. $6 \mathrm{Http} / /$ youtu.be/

"La RivoluzioneRussa”ep.7 Http// youtu.be/

"Arrivano gli Yankees" ep. $8 \mathrm{Http} / /$ youtu.be/ EJ9Iy6GuaTk

"La guerra nei cieli" ep 9. Http// youtu.be/ ZSECCGzjk6k

"Caporetto, il destino é sul Piave" ep. $10 \mathrm{Http//} \mathrm{youtu.be/} \mathrm{oRrWLq8eFdU}$

La Primera Guerra Mundial en Color (2003), Simon Berthon. Nugus/Martin Production Ltd.

(1/6). "Catástrofe" Http// youtu.be/ Uhq17xExIWE

(2/6). "Matanza en las trincheras" Http// youtu.be/ IPJ1oBdbByO

(3/6). "Sangre en el aire" Http// youtu.be/ Zz2TPyyQ2GQ

(4/6). "Asesinos en el Mar" Http// youtu.be/ fhoXr8QZ-s

(5/6). "Caos en el Frente Oriental" Http// youtu.be/ Mg1IWOTdffc

(6/6). "Victoria y desesperación" Http// youtu.be/ 9dmq6j4G7O

La Primera Guerra Mundial (2003), Hew Strachan. Hamilton Film Production.

Http// youtu.be/ UHunlqyaYpo?list= PL8ju9xKEsFPB9aR4Q

Capítulo 1." A las Armas"

Capítulo 2. "A la sombra del Águila"

Capítulo 3. "Guerra Global"

Capítulo 4. "Jihad"

Capítulo 5." Alianza mortal"

Capítulo 6. "Fin del Asedio"

Capítulo 7. "El Bloqueo"

Capítulo 8."Revolución"

Capítulo 9. "Última Ofensiva Alemana"

Capítulo 10. "Guerra sin Fin"

The History Channel .'La Tregua de Navidad. La Primera Guerra Mundial” (Documental) Http//youtu.be/ JDSSSGZd3fA

The History Channel.'La jidah del Kaiser" Documental. Http// youtu.be/ StjtX009Zio. 


\section{Conferencias}

Ager, Jon "Science and the First World War" on June $26^{\text {th }} 2014$, (Conference), UCL Lunch Hour Lectures. Http//youtu.be/ oA1hMahtSKQ

Boardman, Terry "Belgian Neutrality and the British Decision for War" on April $28^{\text {th }} 2014$ (Conference), Brussels. Http//youtu.be/6KbseZkdM3E

Boardman, Terry "Britain's Responsability in 1914 for WWI." (Conference), Http//youtu.be /K1yJOzID_xw

Hirschfield, Gerhard "Das historische Erde der ErstenWeltkriegs" Studium Generale:

(1. Conference), Http//youtu.be/ 1-IR-58bo3c? list=

Kammash, Gudrun "Der Konflicktzwischen Clara Immerwahr und Fritz Haber"

Studium Generale: (4. Conference), Http//youtu.be/ YIrCXCUI9RI? list =

Royal Geographical Society"Britain Should Not have Fought in WWI" on April $15^{\text {th }} 2014$ (conference), in the Royal Geographical Society.

\section{Cuadro I}

\section{La Carrera de Armamentos. Gasto militar de las grandes potencias*}

(en millones de libras esterlinas)

\begin{tabular}{|l|c|c|}
\hline & Gasto Militar & TMC \% \\
\hline 1880 & 132 & \\
\hline 1890 & 158 & 1.81 \\
\hline 1900 & 205 & 2.64 \\
\hline 1910 & 288 & 3.46 \\
\hline 1914 & 397 & 3.26 \\
\hline
\end{tabular}

* Alemania, Austria-Hungría, Italia, R.U. Francia y Rusia Elaboración propia con datos de Hobsbawn 2013.

\section{Cuadro 2}

Las Armadas de las principales potencias europeas

(en número de barcos de guerra)

\begin{tabular}{|l|c|c|}
\hline & 1900 & 1914 \\
\hline Gran Bretaña & 49 & 64 \\
\hline Alemania & 14 & 40 \\
\hline Francia & 23 & 28 \\
\hline Austria-Hungría & 6 & 16 \\
\hline Rusia & 16 & 23 \\
\hline
\end{tabular}




\section{Cuadro 3}

\section{Los Ejércitos de las principales potencias europeas}

\begin{tabular}{|l|c|c|c|c|}
\hline \multicolumn{4}{|c|}{ (en millares) } \\
\hline & En Tiempo de paz & Movilizados & En Tiempo de paz & Movilizados \\
\hline Gran Bretaña & 136 & 600 & 160 & 700 \\
\hline India & 200 & & 249 & 3000 \\
\hline Austria-Hungría & 267 & 772 & 800 & 3500 \\
\hline Francia & 503 & 1000 & 1200 & 3800 \\
\hline Alemania & 419 & 1300 & 2200 & 4400 \\
\hline Rusia & 766 & 1213 & 1400 & \\
\hline
\end{tabular}

Datos de Hobsbawn 2013.

\section{Cuadro 4}

Transporte Marítimo: tonelaje de barcos de más de 100 toneladas únicamente

\begin{tabular}{|c|c|c|c|c|}
\hline & \multicolumn{2}{|c|}{ Años } & \multicolumn{2}{|c|}{$\%$} \\
\hline & 1881 & 1913 & 1881 & 1913 \\
\hline Total Mundial & 18325 & 46970 & 100 & \\
\hline Gran Bretaña & 7010 & 18696 & 38 & \\
\hline Estados Unidos & 2370 & 5429 & 13 & \\
\hline Noruega & 1460 & 2458 & 8 & \\
\hline Alemania & 1150 & 5082 & 6 & \\
\hline Italia & 1070 & 1522 & 6 & \\
\hline Canadá & 1140 & 1735 & 6 & \\
\hline Francia & 840 & 2201 & 5 & \\
\hline Suecia & 470 & 1047 & 3 & \\
\hline España & 450 & 841 & 2 & \\
\hline Países Bajos & 420 & 1310 & 2 & \\
\hline Grecia & 330 & 723 & 2 & \\
\hline Dinamarca & 230 & 762 & 1 & \\
\hline Austria-Hungría & 290 & 1011 & 2 & \\
\hline Rusia & 740 & 974 & 4 & \\
\hline Otros & 355 & 3179 & 2 & \\
\hline
\end{tabular}

Elaboración propia con datos de Hobsbawn 2013. 


\section{Cuadro 5}

\section{Tropas Reclutadas del imperio británico por nacionalidad}

\begin{tabular}{|c|c|c|}
\hline & Efectivos & $\%$ \\
\hline Tropas del R.U. en Agosto de 1914 & 733514 & 9.0 \\
\hline \multicolumn{3}{|c|}{ Nuevos reclutamientos para la Guerra } \\
\hline Inglaterra & 4006158 & 48.9 \\
\hline Escocia & 557618 & 6.8 \\
\hline Gales & 272924 & 3.3 \\
\hline Irlanda & 134202 & 1.6 \\
\hline Total Reino Unido & 4970902 & 60.7 \\
\hline Canadá & 418035 & 5.1 \\
\hline Australia y Tasmania & 330000 & 4.0 \\
\hline Nueva Zelanda & 100471 & 1.2 \\
\hline Sudáfrica & 74196 & 0.9 \\
\hline Otros & 43210 & 0.5 \\
\hline Total Dominios & 965912 & 11.8 \\
\hline Tropas Hindús y de Color & 1524187 & 18.6 \\
\hline
\end{tabular}

Elaboración propia con datos de British Army Statistics of the Great War. 